\title{
Kangaroo mother care for low birthweight infants: a randomized controlled trial in different settings
}

\author{
A Cattaneo ${ }^{1}, \mathrm{R}_{\text {Davanzo }}{ }^{1}$, B Worku ${ }^{2}$, A Surjono $^{3}$, M Echeverria $^{4}$, A Bedri ${ }^{2}$, E Haksari $^{3}$, L Osorno ${ }^{4}$, B Gudetta ${ }^{2}$, \\ D Setyowireni $^{3}$, S Quintero ${ }^{1}$, G Tamburlini $^{1}$ \\ Bureau for International Health ${ }^{1}$, Istituto per l'Infanzia, Trieste, Italy, Ethio-Swedish Children Hospital ${ }^{2}$, Addis Ababa, Ethiopia; \\ Dr Sardjito Hospital ${ }^{3}$, Yogyakarta, Indonesia; and Centro Medico Nacional ${ }^{4}$,Merida, Mexico
}

\begin{abstract}
Cattaneo A, Davanzo R, Worku B, Surjono A, Echeverria M, Bedri A, Haksari E, Osorno L, Gudetta B, Setyowireni D, Quintero S, Tamburlini G. Kangaroo mother care for low birthweight infants: a randomized controlled trial in different settings. Acta Pædiatr 1998; 87: 976-85. Stockholm. ISSN 0803-5253

A randomized controlled trial was carried out for $1 \mathrm{y}$ in three tertiary and teaching hospitals, in Addis Ababa (Ethiopia), Yogyakarta (Indonesia) and Merida (Mexico), to study the effectiveness, feasibility, acceptability and cost of kangaroo mother care (KMC) when compared to conventional methods of care (CMC). About $29 \%$ of 649 low birthweight infants (LBWI; 1000-1999 g) died before eligibility. Of the survivors, $38 \%$ were excluded for various reasons, 149 were randomly assigned to KMC (almost exclusive skin-to-skin care after stabilization), and 136 to CMC (warm room or incubator care). There were three deaths in each group and no difference in the incidence of severe disease. Hypothermia was significantly less common in KMC infants in Merida (13.5 vs 31.5 episodes/100 infants/d) and overall (10.8 vs 14.6). Exclusive breastfeeding at discharge was more common in KMC infants in Merida (80\% vs 16\%) and overall (88\% vs $70 \%)$. KMC infants had a higher mean daily weight gain ( $21.3 \mathrm{~g}$ vs $17.7 \mathrm{~g})$ and were discharged earlier (13.4 vs $16.3 \mathrm{~d}$ after enrolment). KMC was considered feasible and presented advantages over CMC in terms of maintenance of equipment. Mothers expressed a clear preference for KMC and health workers found it safe and convenient. KMC was cheaper than CMC in terms of salaries (US\$ 11788 vs US\$29888) and other running costs (US\$ 7501 vs US\$ 9876). This study confirms that hospital KMC for stabilized LBWI 1000$1999 \mathrm{~g}$ is at least as effective and safe as CMC, and shows that it is feasible in different settings, acceptable to mothers of different cultures, and less expensive. Where exclusive breastfeeding is uncommon among LBWI, KMC may bring about an increase in its prevalence and duration, with consequent benefits for health and growth. For hospitals in low-income countries KMC may represent an appropriate use of scarce resources. $\square$ Developing countries, kangaroo mother care, low birthweight infants
\end{abstract}

A Cattaneo, Bureau for International Health, Istituto per l'Infanzia, Via dell'Istria 65/1, 34137 Trieste, Italy

About $98 \%$ of the 5 million neonatal deaths estimated by WHO for 1995 occurred in low-income countries (1); more than two-thirds were early neonatal deaths. Low birthweight is the underlying cause of most of these deaths (2). Globally, about 25 million births/y (17\%) are of low weight, $<2500 \mathrm{~g},>95 \%$ in low-income countries (3). Good quality care of low birthweight infants (LBWI) could reduce neonatal mortality in these countries, but the technologies used in rich countries are too expensive and need often-lacking skilled personnel, maintenance and logistic support. Expensive technologies may also prevent early mother-baby contact and breastfeeding, two components of newborn care that are essential for survival in low-income countries (4), and may not allow mothers to become competent and confident in the care of their LBWI.

Kangaroo mother care (KMC), defined as early, prolonged and continuous skin-to-skin contact between a mother and her newborn LBWI, both in hospital and after early discharge, until at least the 40th week of postnatal gestational age, could help to overcome most of the constraints of conventional methods of care (CMC). $\mathrm{KMC}$ does not need sophisticated equipment, and for its simplicity it can be applied almost everywhere, including peripheral maternity units of very low-income countries. KMC could also contribute to the humanization of neonatal care and the containment of cost; for these features, it may also be attractive for neonatal units in high-income countries. Since its first description in 1983 (5), KMC has drawn the attention of international agencies and the scientific community, leading to the publication of more than 130 papers and abstracts. Most report local experiences or uncontrolled studies. The published controlled trials from industrialized countries (6-9) concern very small samples, with skin-to-skin contact limited to an average of 10-30 $\mathrm{min} / \mathrm{d}$ or $2.5 \mathrm{~h}$ for $1 \mathrm{~d}$ only, and focus primarily on physiologic outcomes. Of the three controlled studies from lowincome countries, one is a two-cohort study comparing ambulatory KMC with hospital CMC (10), the second is a randomized controlled trial (RCT) of KMC and CMC in hospital, with a 6-month follow-up (11), and the third is an RCT of ambulatory KMC vs hospital CMC in stabilized 
LBWI (12). The three studies lend support to the effectiveness and safety of KMC, but do not clarify many questions related to its feasibility, acceptability and cost, i.e. the very factors that will influence the degree of implementation of this method of care. To further explore effectiveness and safety, and to answer the questions regarding feasibility, acceptability and cost, the Bureau for International Health of the Istituto per l'Infanzia in Trieste, Italy, based on previous experience in Mozambique $(13,14)$, developed in 1994 a protocol for a multicentre RCT.

\section{Materials and methods}

The study protocol was accepted by five hospitals, but two, in India and Brazil, were unable to carry out the RCT; they conducted a controlled trial without randomization and an observational study, respectively. The findings of these two studies will be analysed separately. The full RCT protocol was carried out in Addis Ababa, Yogyakarta and Merida, three settings with very different social, economic and health features (Table 1). The type of health care provided by the three hospitals is also different, as shown by the 1995 data of Table 2. In the same year, the birthweight-adjusted mortality rate in the neonatal units was $7.5 \%$ in Addis Ababa, 3.4\% in Yogyakarta, and 1.9\% in Merida; the overall hospital mortality in neonates $1000-1999 \mathrm{~g}$ was $35 \%, 39 \%$ and $24 \%$, respectively.

The LBWI assigned to CMC were offered the routine care of each hospital: in a warm room in Addis Ababa, with open cribs and the possibility of rewarming in a bulbheated cot, and in incubators in the other two hospitals. Skin-to-skin contact with their mothers was not allowed. All mothers stayed in the hospital in Addis Ababa and Yogyakarta, in separate rooms but with access to their babies for breastfeeding, whereas mothers were not allowed to stay in Merida, but could come at any time during the day (not at night) to visit their offspring. The LBWI assigned to KMC stayed in 4-bed rooms with their mothers. They were kept in close and continuous skin-toskin contact, between the breasts, naked except for a diaper and a hat, covered across their backs with their mother's clothes, day and night, for an average of about $20 \mathrm{~h} / \mathrm{d}$, including when the mother was asleep. The mother was occasionally replaced, for few hours, by another personusually the father or a member of the family - when she needed, for various reasons, to be separated from the baby. For very short absences of the mother, $<1 \mathrm{~h}$, the baby was left on the mother's bed, covered by a blanket. The same instructions on feeding and general care of the baby were given to all mothers during admission irrespective of assignment.

To be eligible for the study, a LBWI ought to have: birthweight between 1000 and $1999 \mathrm{~g}$ without gestational age limits, no dependency on oxygen and/or i.v. fluids, ability (at least partial) to feed, no visible major malformation, and mother present and willing to collaborate. At eligibility, infants were randomly assigned to either CMC or KMC after informed consent. The principal investigators in each hospital used a list of random numbers prepared in

Table 1. General information on the study countries and sites.

\begin{tabular}{|c|c|c|c|}
\hline & Addis Ababa (Ethiopia) & Yogyakarta (Indonesia) & Merida (Mexico) \\
\hline IMR/1000 live births (national, 1995) & 107 & 48 & 33 \\
\hline GNP per capita (US\$, 1993) & 100 & 740 & 3610 \\
\hline Minimum monthly salary (US\$, 1995) & 15 & 60 & 145 \\
\hline Literacy in adult men (national, 1990) & $41 \%$ & $88 \%$ & $90 \%$ \\
\hline $\begin{array}{l}\text { Literacy of adult women as a percentage of literacy in adult } \\
\text { men (national, 1990) }\end{array}$ & $51 \%$ & $85 \%$ & $94 \%$ \\
\hline Births assisted by trained professionals (national, 1995) & $14 \%$ & $36 \%$ & $77 \%$ \\
\hline Proportion of LBWI (national, 1995) & $16 \%$ & $14 \%$ & $12 \%$ \\
\hline Climate (local) & cool & hot and humid & hot and humid \\
\hline Local daily temperature (range) & $3-25^{\circ} \mathrm{C}$ & $24-32^{\circ} \mathrm{C}$ & $24-32^{\circ} \mathrm{C}$ \\
\hline Type of hospital & $\begin{array}{l}\text { public, teaching, children } \\
\text { hospital }\end{array}$ & $\begin{array}{l}\text { public, teaching, general } \\
\text { hospital }\end{array}$ & $\begin{array}{l}\text { social security, } \\
\text { teaching, } \mathrm{MCH} \\
\text { hospital }\end{array}$ \\
\hline Payment by patients & $\begin{array}{l}70 \% \text { exempted; } 30 \% \\
\text { pay a subsidized rate of } \\
\text { US } \$ 2 / d\end{array}$ & $\begin{array}{l}\text { all patients have to pay; } \\
\text { but most are covered by } \\
\text { insurance }\end{array}$ & $\begin{array}{l}\text { all patients covered by } \\
\text { social security (one } \\
\text { family member } \\
\text { regularly employed) }\end{array}$ \\
\hline Population served & all social groups & lower middle class & middle class \\
\hline Literacy of female patients & $\begin{array}{l}50 \% \text {; most with lower } \\
\text { education }\end{array}$ & $\begin{array}{l}90 \% \text {; low proportion } \\
\text { of higher education }\end{array}$ & $\begin{array}{l}90 \% ;>50 \% \text { with } \\
\text { higher education }\end{array}$ \\
\hline Unemployment of female patients & $95 \%$ & $70 \%$ & $60 \%$ \\
\hline
\end{tabular}


Table 2. Some health care features of the study hospitals (1995).

\begin{tabular}{|c|c|c|c|}
\hline & Addis Ababa (Ethiopia) & Yogyakarta (Indonesia) & Merida (Mexico) \\
\hline Deliveries per year & 5000 & 2300 & 5000 \\
\hline Caesarean section rate & $14 \%$ & $8 \%$ & $40 \%$ \\
\hline Neonatal admissions & 1000 & 450 & 1750 \\
\hline From other hospitals & $70 \%$ & $45 \%$ & $15 \%$ \\
\hline Less than $2500 \mathrm{~g}$ & $50 \%$ & $80 \%$ & $20 \%$ \\
\hline Doctors in neonatal unit & 8 & 10 & 15 \\
\hline Nurses in neonatal unit & 18 & 14 & 40 \\
\hline Beds in neonatal unit & 32 & 24 & 30 \\
\hline Warm room & $\begin{array}{c}\text { kept at } 32^{\circ} \mathrm{C} \pm 1 \text { with electrical } \\
\text { heaters }\end{array}$ & no & $\begin{array}{l}\text { no (but air conditioning } \\
\text { turned off) }\end{array}$ \\
\hline Radiant warmer & $\begin{array}{c}1 \text { bulb-heated cot for observation } \\
\text { and rewarming }\end{array}$ & 1 & 7 \\
\hline Incubator & 0 & 10 & 12 \\
\hline Transport incubator & 0 & 2 & 3 \\
\hline Water-filled heated mattress & 2 (out of order) & 0 & 1 \\
\hline $\begin{array}{l}\text { Exclusive breastfeeding } \\
\text { (healthy babies) }\end{array}$ & $\begin{array}{l}>90 \% ; \text { (average discharge after } \\
24 \mathrm{~h})\end{array}$ & $\begin{array}{c}\text { almost } 100 \% \text {; (average discharge } \\
\text { after } 3-5 \mathrm{~d})\end{array}$ & $\begin{array}{c}\text { almost } 100 \% \text {; (discharge at } \\
6-8 \mathrm{~h} \text { for healthy babies, at } \\
48-72 \mathrm{~h} \text { for CS) }\end{array}$ \\
\hline Exclusive breastfeeding (LBWI) & almost $100 \%$ & about $80 \%$ & about $15 \%$ \\
\hline
\end{tabular}

advance for this purpose. In Yogyakarta, randomization was carried out in blocks of six and stratifying by weight. The enrolled infants were kept under observation until achievement of the criteria for discharge from hospital: weight at least $1500 \mathrm{~g}$, clear upward growth trend (at least $15 \mathrm{~g} / \mathrm{kg} / \mathrm{d}$ ) and stable temperature (never $<36.5^{\circ} \mathrm{C}$ ) for at least $3 \mathrm{~d}$, satisfactory ability to suck, good general conditions and mother considered capable of good home care. After discharge, infants were followed-up as outpatients at least four times, at 3,10,20 and $30 \mathrm{~d}$, and as usually scheduled in each hospital afterwards. KMC mothers were encouraged to continue with kangaroo care at home after discharge.

The outcome variables for newborn health were episodes of pre-defined serious illness, episodes of hypothermia and hyperthermia, exclusive breastfeeding as defined by WHO (15), and weight gain, both during admission and at followup. Deaths were recorded but the study design would not allow to identify differences in mortality. The main variables for feasibility were the adequacy of the structure (crowding, food, water, bath, toilet), the availability of staff, equipment and supplies (including fuel and electricity), and the need for maintenance and repair. The acceptability outcomes were the feelings of health workers about the presence of mothers and the advantages, disadvantages and safety of each method of care, and those of the mothers about the comfort, convenience and constraints of each method. Mothers and health workers were also asked about their preferred method of care in case of an hypothetical LBW birth. The cost variables included two types of running costs: salaries and other items (food for mothers and babies, laundry and linen, drugs and other medical supplies, X-ray and laboratory, fuel and electricity and maintenance of equipment). The cost of salaries was calculated by estimating the proportion of staff time spent on KMC and $\mathrm{CMC}$ and multiplying it by the number of months worked during the study by different categories of health workers; the other costs by keeping accurate monthly records. Because the study focussed mainly on feasibility, acceptability and cost, it was difficult to determine the appropriate sample size. Considering the difficulties of conducting a trial in different settings of low-income countries, and the financial constraints, it was decided to limit the duration of the study to 12 months and to enrol and randomize about $100 \mathrm{LBWI} /$ site.

The data were collected with six tools: a form to record data on each case during admission, a form for the followup, two semi-structured questionnaires for interviews with mothers and staff, a monthly record of observed constraints, and a monthly form to summarize the cost of each method of care. Each tool was tested and adapted during the preparation phase, during which definitions and procedures were standardized. The two questionnaires were also translated into the local language. The principal investigator in each site was in charge of monitoring the quality of data collection during the whole study period. One person in each site entered data using EpiInfo. Qualitative data from the two questionnaires were coded before data entering. The databases were checked for completeness and errors, and were then analysed in Trieste, during and after a meeting of the principal investigators. Analysis was carried out using ANOVA, $\chi^{2}, t$ - and Kruskal-Wallis tests, stratifying by site, sex, birthweight and socioeconomic variables.

\section{Results}

Table 3 gives the number of LBWI weighing between 1000 and $1999 \mathrm{~g}$ admitted in each hospital during the study 
Table 3. Populations from which the study subjects were enrolled.

\begin{tabular}{|c|c|c|c|c|}
\hline & Addis Ababa (Ethiopia) & Yogyakarta (Indonesia) & Merida (Mexico) & Overall \\
\hline Study period & Sep 95-Aug 96 & Aug 95-Jul 96 & Oct 95-Sep 96 & Aug 95-Sep 96 \\
\hline Admissions $1000-1999 \mathrm{~g}$ & 346 & 172 & 131 & 649 \\
\hline Dead before eligibility & $125(36 \%)$ & $43(25 \%)$ & $18(14 \%)$ & $186(29 \%)$ \\
\hline Remaining babies & 221 & 129 & 113 & 463 \\
\hline Enrolled babies & $100(45 \%)$ & $106(82 \%)$ & $79(70 \%)$ & $285(62 \%)$ \\
\hline Excluded babies & $121(55 \%)$ & $23(18 \%)$ & $34(30 \%)$ & $178(38 \%)$ \\
\hline Reasons for exclusion & $\begin{array}{c}\text { multiple birth } 67 ; \text { mother } \\
\text { not available } 52 ; \\
\text { no consent } 2\end{array}$ & $\begin{array}{l}\text { multiple birth } 14 \text {; mother } \\
\text { not available } 5 ; \\
\text { malformations } 4\end{array}$ & $\begin{array}{c}\text { no consent } 31 \text {; refused } \\
\text { CMC after randomization } 3\end{array}$ & \\
\hline
\end{tabular}

period. About 29\% died during the stabilization phase, before eligibility. A variable proportion was excluded in each site for different reasons. In Addis Ababa, many infants were excluded because their mothers had abandoned them, a common event for LBWI in this setting. Sixty-seven more were excluded because of multiple birth, including eight sets of triplets and one set of six (the EthioSwedish Children Hospital is a national reference centre for multiple pregnancies). Though multiple birth was not among the exclusion criteria, it became so when twins were randomly assigned to a different intervention. In practice, eligible twins were enrolled only if the sibling died. This happened in Yogyakarta as well, where the proportion of excluded babies was the lowest. The proportion was intermediate in Merida, and totally due to mothers who refused to participate before or after random assignment.

Table 4 gives the characteristics of the 285 LBWI eventually randomized. There were few significant differences between groups in each site: a higher proportion of primiparae in the KMC group in Merida, a higher proportion of infants with gestational age $<32$ weeks in the KMC group in Addis Ababa, and a lower proportion of exclusively breastfed babies at admission and enrolment in the CMC group in Yogyakarta. The last difference is responsible for the only overall difference between groups. There were many statistically significant differences among sites: in birthweight, gestational age (GA), weight at admission, and age, weight and breastfeeding at enrolment. The study subjects were observed in the hospitals for a total of 4205 infant/d: 1139 in Addis Ababa, 2010 in Yogyakarta, and 1056 in Merida.

The health outcomes are shown in Table 5. Six deaths were recorded, equally distributed between groups. Twenty $\mathrm{KMC}$ infants had to be transferred to CMC for $1-15 \mathrm{~d}$ for different reasons (severe bacterial infections, IV therapy, oxygen therapy); all returned to $\mathrm{KMC}$ as soon as the problem was solved, except for the three who died. The overall difference in the number of episodes of severe disease is not statistically significant $(\mathrm{RR}=0.63 ; 95 \%$ CI $0.33-1.21 ; p=0.16)$, and was entirely due to 15 cases of severe mucocutaneous infection (conjunctivitis, stomatitis, omphalitis) recorded in Yogyakarta. Hypothermia, an axillary temperature of $<36.0^{\circ} \mathrm{C}$, was significantly more common in CMC infants. The only significant difference was recorded in Merida $(\mathrm{RR}=0.43 ; 95 \% \mathrm{CI}$ $0.33-0.56 ; p=0.00001)$; this difference, added to the smaller differences in the other two hospitals, was responsible for the overall difference $(\mathrm{RR}=0.74 ; 95 \%$ CI $0.62-$ $0.88 ; p=0.0005)$. Hyperthermia, an axillary temperature $>38.5^{\circ} \mathrm{C}$ without other cause, was also more common in CMC infants $(\mathrm{RR}=0.09 ; 95 \%$ CI $0.01-0.72 ; p=0.004)$, but this overall difference was due to the 10 episodes recorded in Yogyakarta. The other differences concerned exclusive breastfeeding, growth and length of stay in the hospital (LOS). KMC babies breastfed more, though the overall result was mainly attributable to Merida, and showed a trend towards higher mean daily weight gain during the study in all sites, though the difference (21.3 vs $17.7 \mathrm{~g} / \mathrm{d}$ ) was significant only for the overall result. For this reason, KMC infants achieved the discharge weight in a shorter time and went home $11 \mathrm{~d}$ after enrolment (median value; mean 13.4) compared with $13 \mathrm{~d}$ (mean 16.3) for $\mathrm{CMC}$ infants. The growth advantage of KMC during the study is lost if growth is calculated from birth; in this case, KMC infants gained $9.1 \mathrm{~g} / \mathrm{d}$ on average compared with $8.2 \mathrm{~g} / \mathrm{d}$ for CMC infants (difference not significant). As for characteristics at enrolment, there were differences among sites also in health outcomes, as shown by the last column of Table 5.

At follow-up, 129/146 (88\%) KMC and 112/133 (84\%) CMC babies, proportionally distributed among the 3 hospitals, attended the first visit; the number went down to 93 $(64 \%)$ and $82(62 \%)$, respectively, at the fourth visit. The mean age at each visit was the same in both groups and was higher in Merida than in Addis Ababa and Yogyakarta. The relative loss to follow-up was the same by group and by site. None of the studied variables revealed a difference, except for the proportion of exclusively breastfed babies. This was always higher in KMC than in CMC infants: from $111 / 129(86 \%)$ vs $94 / 112(84 \%)$ at first follow-up, to $73 / 93$ $(78 \%)$ vs $59 / 82(72 \%)$ at fourth. The only significant difference was recorded at the third follow-up visit: $86 /$ $104(83 \%)$ vs $64 / 92(70 \%)$, respectively $(p=0.03)$, mainly because of a difference in Yogyakarta (43/43, $100 \%$, vs $34 / 42,81 \%$; $p=0.002$ ).

In Addis Ababa there was insufficient room for CMC mothers and infants, owing to overcrowding, $60 \%$ of the time; the 4-bed room set up for KMC was sufficient and felt 


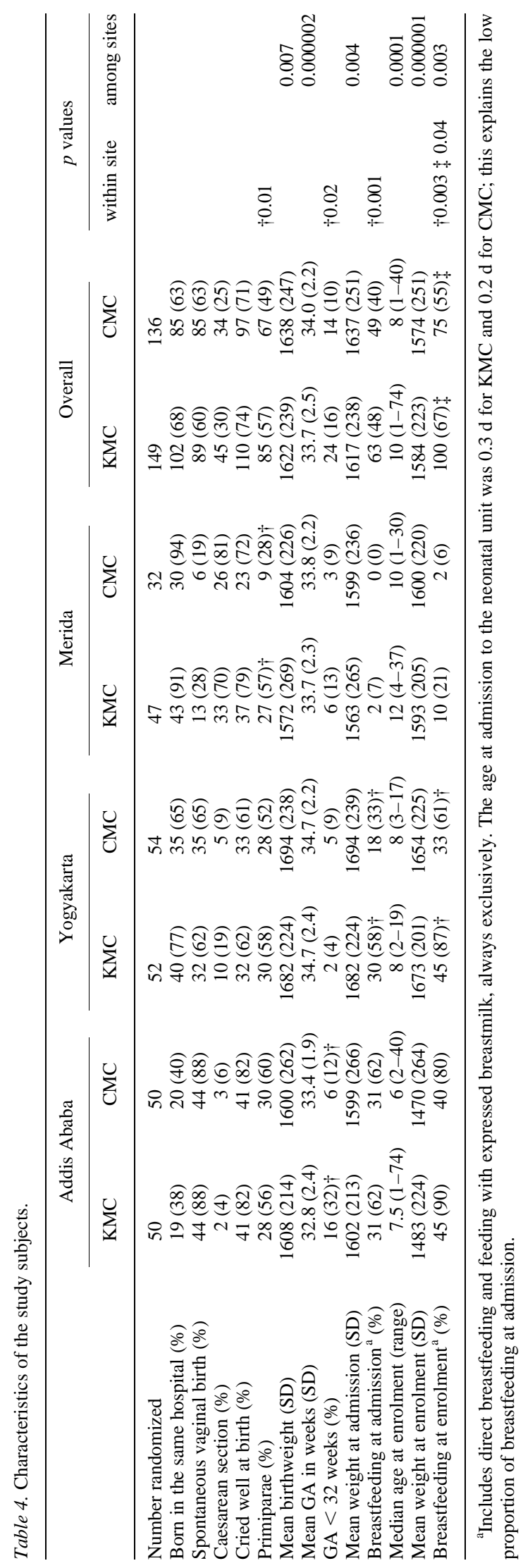




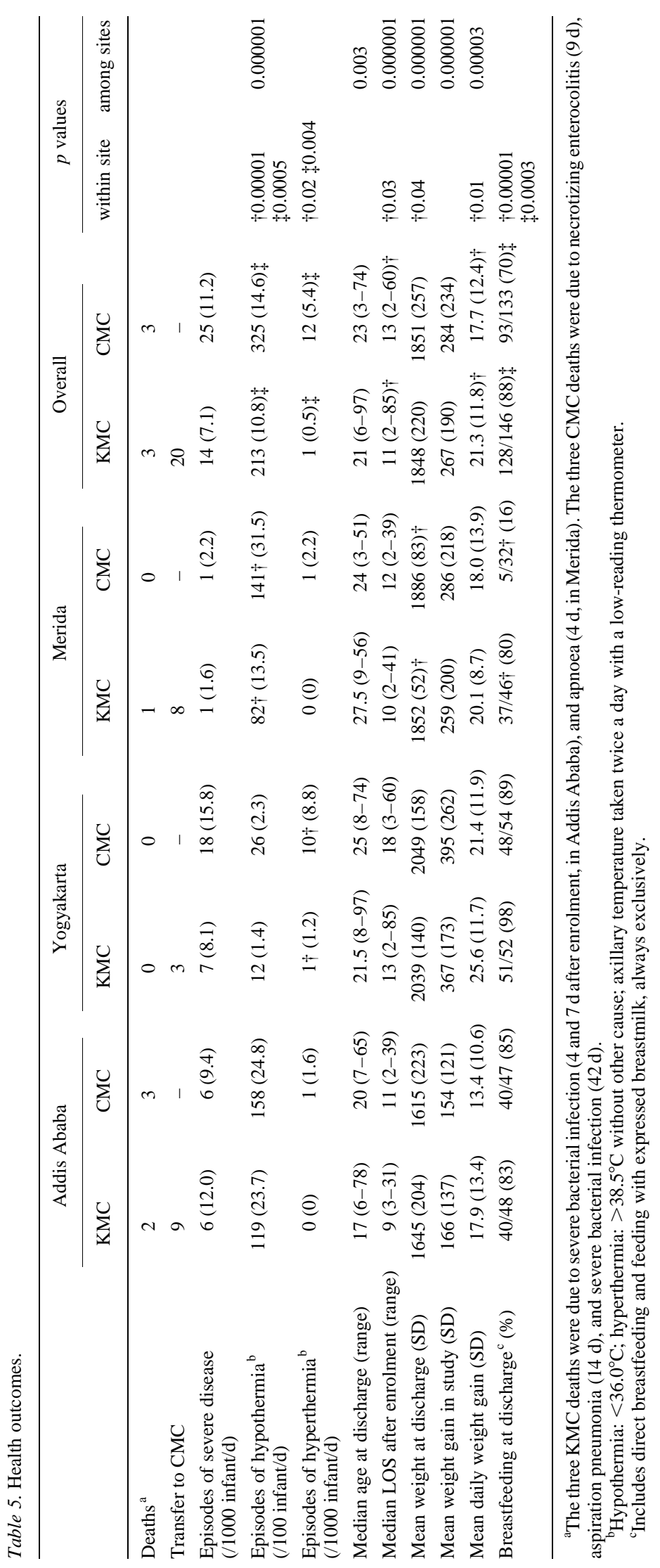




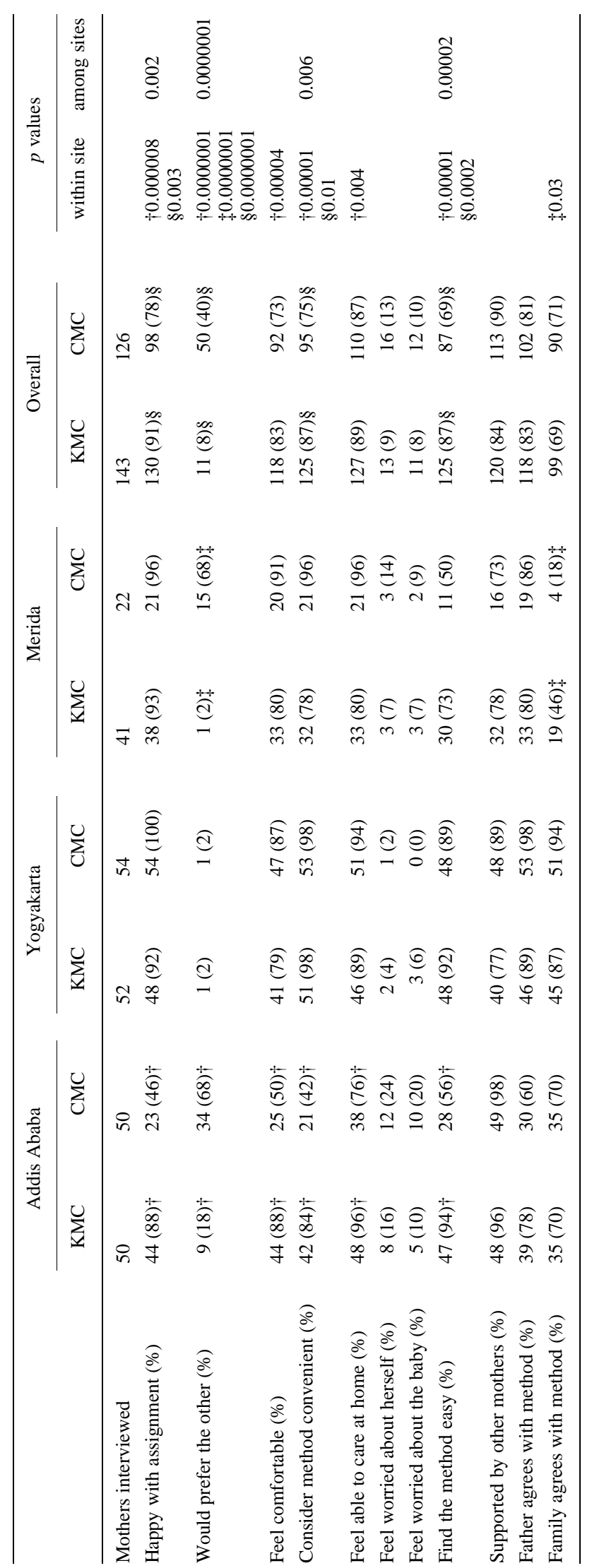


to be comfortable by the mothers. The CMC ward had an insufficient number of cots and infants had to share the same cot sometimes. Cooking and eating facilities were inadequate for both groups. All the necessary materials were always available, except for drugs, completely available only $20 \%$ of the time, and oxygen, $80 \%$ of time. The CMC thermal control system was always working well, but had to undergo maintenance or repair in 7/12 months. Outbreaks of infection were recorded once in the KMC and four times in the CMC ward. In Yogyakarta, both the KMC and CMC wards had no problems, with food, drugs, materials, supplies, electricity, and maintenance; no outbreak of infection was recorded. In Merida, the CMC ward was inadequate during visiting hours owing to overcrowding. The KMC ward was adequate, but the hospital had to provide extra food, linen and laundry. The staff was insufficient to deal with both the CMC and KMC wards. There was no problem with materials and supplies, including drugs, but some lack of food and linen for KMC mothers. There were some problems with thermal control and maintenance of equipment in the CMC ward, about one-third of the time, but solutions were always found. One outbreak of infection was recorded in the KMC ward during the study.

Interviews with the staff took place in the second half of the study; 49 health workers ( 15 doctors, 31 nurses, and 3 other professionals) were interviewed, representing $45 \%$, proportionally distributed among sites, of the professional staff engaged in the study. Most $(82 \%)$ were female with a mean age of $36 y$ (range 22-52), and $88 \%$ had children. They had worked for an average of $3.4 \mathrm{y}$ in the same ward, attending about 23 patients per shift (13 in Merida and 35 in Addis Ababa), working $47 \mathrm{~h} /$ week on average. Ninety-two percent considered KMC safe, and $90 \%$ found that mothers were comfortable with it compared with $69 \%$ for CMC. When asked whether they would prefer KMC or CMC if they had a LBWI, 100\% chose KMC in Addis Ababa and Merida, compared with $41 \%$ in Yogyakarta, where $6 \%$ chose CMC and $47 \%$ were uncertain.

Almost all mothers (100\% in the KMC and $98 \%$ in the CMC group) were interviewed, on average $10.7 \mathrm{~d}$ after enrolment. There were no differences between groups in mean age (26.3 y), reproductive history, education, social and economic conditions. The mean number of years of education was lower in Addis Ababa and higher in Yogyakarta and Merida, while the opposite was true for the proportion of unemployed women and partners, as expected. The only difference between groups regarded the availability of running water at home, higher in KMC than in CMC mothers ( $84 \%$ vs $73 \%$; $p=0.03$ ), entirely due to a difference in Yogyakarta. Ninety-eight percent of mothers stated that they had been fully informed about the study and $91 \%$ were satisfied with the information received about the two methods of care. Table 6 presents some results of these interviews and shows a clear preference for KMC. About $11 \%$ of the KMC mothers complained about the long stay in the hospital; conversely, $98 \%$ of the CMC mothers would have liked to spend more time with their babies. Some KMC mothers (13\% on average, but $29 \%$ in Addis Ababa) were worried about the care of their other children left at home, almost always looked after by the father or a member of the family, but the worry was partially relieved by the daily or very frequent visits most of them (93\%) received while in hospital.

The running cost was about $50 \%$ less for KMC. Specifically, it was US\$ 11788 vs US\$ 29888 for salaries, and US\$ 7501 vs US\$ 9876 for other items, overall. The cost for salaries was low because the average monthly salaries of doctors, nurses and other staff were US\$ 250, 96 and 55, respectively. Among the running cost for other items, electricity was much higher for $\mathrm{CMC}$, mainly due to the cost of keeping the ward at $32^{\circ} \mathrm{C}$ in Addis Ababa, and maintenance was much higher for CMC everywhere. The only items for which KMC was more expensive than CMC were food for mothers and laundry, almost totally due to the expenses met by the hospital in Merida.

\section{Discussion}

Conducting RCTs in developing countries is difficult but important (16); it is particularly difficult to run an effectiveness, i.e. a study that includes feasibility, acceptability and cost, rather than an efficacy trial, as recommended by Clemens for vaccine trials (17). The risk of failure is high: only 5 hospitals, out of the 11 to which the protocol was proposed, eventually agreed to conduct the study and only 3 successfully completed the RCT. This trial was particularly problematic because it went beyond biomedical measurements to investigate managerial, financial and cultural issues. The many differences among sites make the overall comparison of $\mathrm{KMC}$ and $\mathrm{CMC}$ problematic: it provides just a general indication that should not be interpreted without continuous reference to the specific features of the study population and the findings of each site. Moreover, $38 \%$ of eligible LBWI were excluded; many of them were twins, and perhaps a different randomization strategy could have reduced this problem. Despite these difficulties, the results are important because they complement the evidence provided by previous studies.

As previously reported $(11,12)$, the majority of deaths in LBWI occur before stabilization. This happened in our study as well. Hospital-based KMC after stabilization is therefore unlikely to contribute to a reduction of mortality. The only study that has so far demonstrated a mortality reduction comes from a small mission hospital in Zimbabwe, where KMC was applied at birth (18); however, this was a study using a historical control group, not a RCT. More controlled studies are needed on the effectiveness and safety of KMC before stabilization in secondary and primary units of low-income countries, where good care for LBWI is rarely available. Ambulatory KMC after stabilization, as opposed to an inpatient minimal care unit, seems also safe in terms of mortality as shown by the two studies from Colombia $(10,12)$. KMC may also bring about some advantages in terms of serious morbidity, as reported from Ecuador (11). The potential long-term benefits of KMC 
related to prolonged breastfeeding, better bonding, and greater ability of the mother to deal with a LBWI, have not been studied yet.

Our study recorded a better growth and a higher proportion of exclusive breastfeeding among KMC infants. The advantage of KMC for growth occurred only in hospital and after enrolment; it disappears when total growth, from birth to follow-up, is considered. The KMC literature is not consistent on feeding and growth. While most studies in settings where the prevalence of breastfeeding in LBWI is low show an increased prevalence and duration in KMC infants $(6,19-21)$, with a consequent benefit on growth, reports from settings where breastfeeding is common tend to show no difference $(11,12)$. The likely conclusion is that breastfeeding is the important factor: as far as $\mathrm{KMC}$ promotes and stimulates more exclusive breastfeeding, when compared to CMC, it is also beneficial for growth. Alternative explanations include possible effects on growth of better thermal regulation and reduced levels of infant stress.

Bergman and Jürisoo (18) state that $\mathrm{KMC}$ is not only feasible, but also easily grasped by the hospital staff and accepted by the community. The feasibility of KMC is also testified by the growing number of reported experiences and by its inclusion in national guidelines for perinatal care (in Mozambique and Indonesia, for example). Our monthly recording of constraints showed that $\mathrm{KMC}$ is more feasible than CMC where mothers are already admitted to hospital with their LBWI. CMC needs more space, is less comfortable for patients and staff, depends on a constant supply of electricity and oxygen which is far from being granted in most low-income countries, and has more maintenance problems. On the other hand, KMC may present problems where mothers are not usually staying in hospital for CMC: if mothers are to stay, then more rooms with beds are needed for them, as well as food and laundry.

Our study shows that KMC is more acceptable than $\mathrm{CMC}$ for staff and mothers. A significantly higher proportion of health workers would prefer KMC if they had a LBWI, and more mothers would switch from CMC to KMC than vice versa. KMC seems to be acceptable in different cultures and environments, though continuous skin-to-skin contact may be uncomfortable in hot and humid countries. Though part of our results on acceptability may be influenced by the 4-bed rooms reserved for KMC and contaminated by the closeness of the KMC and CMC wards, the difference seems so striking in favour of KMC, except for Yogyakarta, to leave no doubt about what mothers prefer-to stay with their babies-as shown also by the three mothers, withdrawn from the study, who refused CMC and opted for KMC in Merida after informed consent and random assignment to the former.

Only the controlled trial in Ecuador (11), in addition to ours, looked at cost so far. In Quito, the cost of hospital care was lower for $\mathrm{KMC}$, due to the use of incubators in CMC. $\mathrm{KMC}$ was cheaper also after discharge, but the follow-up cost data were gathered only on 49 subjects out of 275 initially enrolled. The direct cost for the mothers, however, was higher for the $\mathrm{KMC}$ group, owing to the greater number of clinic visits; this may not be a disadvantage and may in fact be an explanation for the lower incidence of serious illness reported in KMC infants. Our trial confirms that $\mathrm{KMC}$ is cheaper than $\mathrm{CMC}$ in terms of direct cost. The savings in running cost derive from the small but important reduction in length of stay (a benefit in itself irrespective of cost), from the lower proportion of staff time devoted to $\mathrm{KMC}$ (this is not a real saving for the hospital, as salaries are paid anyway; it is a saving in the sense that health professionals can perform other tasks), and from the lower need for oxygen, fuel, electricity and maintenance. KMC can cost more only where CMC mothers are not allowed to stay in the hospital. Capital cost is probably much less for $\mathrm{KMC}$ in terms of equipment.

Our study confirms that hospital KMC for stabilized LBWI $1000-1999 \mathrm{~g}$ is at least as effective and safe as $\mathrm{CMC}$, and shows that it is feasible, acceptable, and less expensive. For these features, KMC may be preferred to $\mathrm{CMC}$ in settings with very scarce resources; this is also the recommendation of a group of $\mathrm{KMC}$ researchers who set up the International Network on KMC in $1996(22,23)$. There is no evidence so far, despite the many reports of better physiologic performance, that $\mathrm{KMC}$ is more effective than $\mathrm{CMC}$ in terms of mortality. KMC might be associated with some reduction of morbidity, and there might be some advantages for nutrition in settings where exclusive breastfeeding for LBWI is uncommon.

Acknowledgments. - This study was supported by a Research Grant of the Ministry of Health of Italy and by the Division of Reproductive Health (Technical Support) of the World Health Organization, Geneva, Switzerland (Contract M3/181/289).

\section{References}

1. WHO. Perinatal mortality. A listing of available information. Maternal Health and Safe Motherhood Programme (WHO/FRH/MSM/ 96.7), Geneva, 1996

2. WHO. Essential newborn care. Report of a Technical Working Group, Trieste, 25-29 April 1994. Maternal and Newborn Health/ Safe Motherhood (WHO/FRH/MSM/96.13), Geneva, 1996

3. WHO. Low birth weight. A tabulation of available information. Maternal Health and Safe Motherhood Programme (WHO/MCH/ 92.2), Geneva, 1992

4. WHO. Mother-baby package: implementing safe motherhood in countries. Maternal Health and Safe Motherhood Programme (WHO/FHE/MSM/94.11), Geneva, 1994

5. Rey ES, Martinez HG. Manejo racional del niño prematuro. In: Curso de Medicina Fetal, Universidad Nacional, Bogotà, Colombia, 1983

6. Whitelaw A, Heisterkamp G, Sleath K, Acolet D, Richards M. Skinto-skin contact for very low birth weight infants and their mothers. Arch Dis Child 1988; 63: 1377-81

7. Ludington-Hoe SM, Thompson CA, Swinth J, Hadeed AJ, Anderson GC. Kangaroo care: research results and practice implications and guidelines. Neonatal Network 1994; 13: 19-25

8. Bier JB, Ferguson AE, Morales Y, Liebling JA, Archer D, Oh W, Vohr B. Comparison of skin-to-skin contact with standard contact in low birth weight infants who are breastfed. Arch Pediatr Adolesc Med 1996; 150: 1265-9

9. Bosque EM, Brady JP, Affonso D, Wahlberg V. Physiologic measures of kangaroo versus incubator care in a tertiary level nursery. J Obstet Gynecol Neonatal Nurs 1995; 24: 219-28 
10. Charpak N, Ruiz-Pelaez JG, Charpak Y. Rey-Martinez kangaroomother programme: an alternative way of caring for low birth weight infants? One year mortality in a two-cohort study. Pediatrics 1994; 94 : 804-10

11. Sloan NL, Leon Camacho LW, Pinto Rojas E, Stern C, and Maternidad Isidro Ayora Study Team. Kangaroo mother method: randomised controlled trial of an alternative method of care for stabilised lowbirthweight infants. Lancet 1994; 344: 782-5

12. Charpak N, Ruiz-Pelaez JG, Figueroa de Calume Z, Charpak Y Kangaroo mother versus traditional care for newborn infants $\leq$ 2000 grams: a randomized controlled trial. Pediatrics 1997; 100 $682-8$

13. Colonna F, Uxa F, da Graça AMP, de Vonderweid U. The "kangaroomother" method: evaluation of an alternative model for the care of low birth weight newborns in developing countries. Int J Gynaecol Obstet 1990; 31: 335-9

14. Davanzo R. Care of the low birth weight infants with the kangaroo mother method in developing countries. In: Proceedings of the First International Conference on the Kangaroo Mother Program, Bogotà Colombia, November 1990. New York: UNICEF, 1992: 451-74

15. WHO. Indicators for assessing breastfeeding practices. Division of Diarrhoeal and Acute Respiratory Disease Control (WHO/CDD/SER/ 91.14), Geneva, 1991

16. Mabey D. Importance of clinical trials in developing countries Lancet 1996; 348: 1113-4

17. Clemens J, Brenner R, Rao M, Tafari N, Lowe C. Evaluating new vaccines for developing countries: efficacy or effectiveness? JAMA 1996; 275: 390-7

18. Bergman NJ, Jürisoo LA. The "kangaroo-method" for treating low birth weight babies in a developing country. Tropical Doctor 1994; 24: 57-60

19. Wahlberg V, Affonso D, Persson B. A retrospective, comparative study using the kangaroo method as a complement to the standard incubator care. Eur J Public Health 1992; 2: 34-7

20. Schmidt E, Wittreich G. Care of the abnormal newborn: a random controlled trial study of the "kangaroo method" of care of low birt weight newborns. In: WHO. Consensus Conference on Appropriate Technology following Birth, Trieste, 7-11 October 1986

21. Syfrett EB, Anderson GC, Behnke M, Neu J. Early and virtually continuous kangaroo care for lower-risk preterm infants: effect on temperature, breastfeeding, supplementation and weight. In: Proceedings of the Biennial Conference of the Council of Nurse Researchers, Washington, DC: American Nurses Association, November 1993

22. Cattaneo A, Davanzo R, Bergman N, Charpak N (on behalf of the International Network on Kangaroo Mother Care). Kangaroo mother care in low-income countries. J Trop Pediatr (in press)

23. Cattaneo A, Davanzo R, Uxa F, Tamburlini G (for the International Network on Kangaroo Mother Care). Recommendations for the implementation of kangaroo mother care for low birth weight infants. Acta Paediatr 1998; 87: 440-5

Received Oct. 27, 1997. Accepted in revised form Apr. 22, 1998 